\title{
ON THE $L_{w}^{2}$-BOUNDEDNESS OF SOLUTIONS FOR PRODUCTS OF QUASI-INTEGRO DIFFERENTIAL EQUATIONS
}

\author{
SOBHY EL-SAYED IBRAHIM
}

Received 14 August 2001

\begin{abstract}
Given a general quasidifferential expressions $\tau_{1}, \tau_{2}, \ldots, \tau_{n}$ each of order $n$ with complex coefficients and their formal adjoints are $\tau_{1}^{+}, \tau_{2}^{+}, \ldots, \tau_{n}^{+}$on $[0, b)$, respectively, we show under suitable conditions on the function $F$ that all solutions of the product of the quasi-integrodifferential equation $\left[\prod_{j=1}^{n} \tau_{j}\right] y=w F\left(t, y, \int_{0}^{t} g(t, s\right.$, $\left.\left.y, y^{\prime}, \ldots, y^{\left(n^{2}-1\right)}(s)\right) d s\right)$ on $[0, b), 0<b \leq \infty ; t, s \geq 0$, are bounded and $L_{w}^{2}$-bounded on $[0, b)$. These results are extensions of those by the author (1994), Wong (1975), Yang (1984), and Zettl $(1970,1975)$.
\end{abstract}

2000 Mathematics Subject Classification: 34A05, 34C11, 34E15, 34G10, 47E05.

1. Introduction. In $[8,11,15]$ Wong and Zettl proved that all solutions of a perturbed linear differential equation belong to $L^{2}(0, \infty)$ assuming the fact that all solutions of the unperturbed equation possess the same property. In [6] the author extends their results for a general quasidifferential expression $\tau$ of arbitrary order $n$ with complex coefficients, and considered the property of boundedness of solutions of a general quasidifferential equation $\tau[y]-$ $\lambda w y=w f(t, y)$, where $\lambda \in \mathbb{C}$, on $[0, b), f(t, s)$ satisfies

$$
|f(t, y)| \leq e_{1}(t)+r_{1}(t)|y|^{\sigma}, \quad t \in[0, b) \text { for some } \sigma \in[0,1],
$$

where $e_{1}(t)$ and $r_{1}(t)$ are nonnegative continuous functions on $[0, b)$.

Our objective in this paper is to extend the results in $[4,6,8,9,11,15]$ to more general class of quasi-integrodifferential equation in the form

$$
\left[\prod_{j=1}^{n} \tau_{j}\right] y=w F\left(t, y, \int_{0}^{t} g\left(t, s, y, y^{\prime}, \ldots, y^{\left(n^{2}-1\right)}(s)\right) d s\right) \quad \text { on }[0, b)
$$

where $0<b \leq \infty ; t, s \geq 0$. Also, we prove under suitable condition on the function $F$ that, if all solutions of the equations $\left(\prod_{j=1}^{n} \tau_{j}\right) y=0$ and $\left(\prod_{j=1}^{n} \tau_{j}^{+}\right) z=0$ belong to $L_{w}^{2}(0, b)$, then all solutions of (1.2) also belong to $L_{w}^{2}(0, b)$, where $\tau_{j}^{+}$ is the formal adjoint of $\tau_{j}, j=1,2, \ldots, n$. 
We deal throughout this paper with a quasidifferential expression $\tau_{j}$ each of arbitrary order $n$ defined by Shin-Zettl matrices (see $[4,13]$ ) on the interval $I=[0, b)$. The left-hand end point of $I$ is assumed to be regular but the righthand end point may be regular or singular.

2. Notation and preliminaries. The domain and range of a linear operator $T$ acting in a Hilbert space $H$ will be denoted by $D(T)$ and $R(T)$, respectively and $N(T)$ will denote its null space. The nullity of $T$, written $\operatorname{nul}(T)$, is the dimension of $N(T)$ and the deficiency of $T$, written $\operatorname{def}(T)$, is the codimension of $R(T)$ in $H$; thus if $T$ is densely defined and $R(T)$ is closed, then $\operatorname{def}(T)=$ $\operatorname{nul}\left(T^{*}\right)$. The Fredholm domain of $T$ is (in the notation of [2]) the open subset $\triangle_{3}(T)$ of $\mathbb{C}$ consisting of those values of $\lambda \in \mathbb{C}$ which are such that $(T-\lambda I)$ is a Fredholm operator, where $I$ is the identity operator in $H$. Thus $\lambda \in \triangle_{3}(T)$ if and only if $(T-\lambda I)$ has a closed range and a finite nullity and deficiency.

A closed operator $A$ in a Hilbert space $H$ has property $(C)$, if it has closed range and $\lambda=0$ is not an eigenvalue, that is, there is some positive number $r$ such that $\|A x\| \geq r\|x\|$ for all $x \in D(A)$.

Note that, property $(C)$ is equivalent to $\lambda=0$ being a regular type point of $A$. This in turn is equivalent to the existence of $A^{-1}$ as a bounded operator on the range of $A$ (which need not be all of $H$ ).

Given two operators $A$ and $B$, both acting in a Hilbert space $H$, we wish to consider the product operator $A B$. This is defined as follows

$$
D(A B):=\{x \in D(B) \mid B x \in D(A)\}, \quad(A B) x=A(B x), \quad \forall x \in D(A B) .
$$

It may happen in general that $D(A B)$ contains only the null element of $H$. However, in the case of many differential operators, the domains of the product will be dense in $H$.

The next result gives conditions under which the deficiency of a product is the sum of the deficiencies of the factors.

LEMMA 2.1 (cf. [4, Theorem A] and [12]). Let $A$ and $B$ be closed operators with dense domains in a Hilbert space $H$. Suppose that $\lambda=0$ is a regular type point for both operators and $\operatorname{def} A$ and $\operatorname{def} B$ are finite. Then $A B$ is a closed operator with dense domain, has $\lambda=0$ as a regular type point and

$$
\operatorname{def} A B=\operatorname{def} A+\operatorname{def} B .
$$

Evidently, Lemma 2.1 extends to the product of any finite number of operators $A_{1}, A_{2}, \ldots, A_{n}$.

We now turn to the quasidifferential expressions defined in terms of a ShinZettl matrix $F$ on an interval $I$. The set $Z_{n}(I)$ of Shin-Zettl matrices on $I$ consists of $n \times n$ matrices $P=\left\{p_{r s}\right\}, 1 \leq r, s \leq n$, whose entries are complex-valued 
functions on $I$ which satisfy the following conditions:

$$
\begin{aligned}
& p_{r s} \in L_{\text {loc }}^{1}(I) \quad(1 \leq r, s \leq n, n \geq 2), \\
& p_{r s} \neq 0 \quad \text { a.e. on } I(1 \leq r \leq n-1), \\
& p_{r s}=0 \quad \text { a.e. on } I(2 \leq r+1<s \leq n) .
\end{aligned}
$$

For $P \in Z_{n}(I)$, the quasiderivatives associated with $P$ are defined by

$$
\begin{aligned}
& y^{[0]}:=y, \\
& y^{[r]}:=\left(p_{r, r+1}\right)^{-1}\left\{\left(y^{[r-1]}\right)^{\prime}-\sum_{s=1}^{r} p_{r s} y^{[s-1]}\right\} \quad(1 \leq r \leq n-1), \\
& y^{[n]}:=\left(y^{[n-1]}\right)^{\prime}-\sum_{s=1}^{n} p_{n s} y^{[s-1]},
\end{aligned}
$$

where the prime ' denotes differentiation.

The quasidifferential expression $\tau$ associated with $P$ is given by

$$
\tau[y]:=i^{n} y^{[n]} \quad(n \geq 2),
$$

this being defined on the set

$$
V(\tau):=\left\{y: y^{[r-1]} \in A C_{\mathrm{loc}}(I), r=1, \ldots, n\right\}
$$

where $L_{\text {loc }}^{1}(I)$ and $A C_{\text {loc }}(I)$ denote, respectively, the spaces of complex-valued Lebesgue measurable functions on $I$ which are locally integrable and locally absolutely continuous on every compact subinterval of $I$.

The formal adjoint $\tau^{+}$of $\tau$ defined by the matrix $P^{+} \in Z_{n}(I)$ is given by

$$
\begin{aligned}
\tau^{+}[z] & :=i^{n} z^{[n]} \quad \forall y \in V\left(\tau^{+}\right), \\
V\left(\tau^{+}\right) & :=\left\{z: z_{+}^{[r-1]} \in A C_{\mathrm{loc}}(I), r=1, \ldots, n\right\},
\end{aligned}
$$

where $z_{+}^{[r-1]}, r=1,2, \ldots, n$, are the quasiderivatives associated with the matrix $P^{+}$,

$$
P^{+}=\left\{p_{r s}^{+}\right\}=(-1)^{r+s+1} \bar{p}_{n-s+1, n-r+1} \quad \text { for each } r, s ; 1 \leq r, s \leq n .
$$

Note that $\left(P^{+}\right)^{+}=P$ and so $\left(\tau^{+}\right)^{+}=\tau$. We refer to $[2,3,6,7,13]$ for a full account of the above and subsequent results on quasidifferential expressions.

Let the interval $I$ have end points $a, b(-\infty \leq a<b \leq \infty)$, and let $w: I \rightarrow \mathbb{R}$ be a nonnegative weight function with $w \in L_{\mathrm{loc}}^{1}(I)$ and $w(x)>0$ (for almost 
all $x \in I$ ). Then $H=L_{w}^{2}(I)$ denotes the Hilbert function space of equivalence classes of Lebesgue measurable functions such that $\int_{I} w|f|^{2}<\infty$; the innerproduct is defined by

$$
(f, g):=\int_{I} w(x) f(x) \overline{g(x)} d x \quad\left(f, g \in L_{w}^{2}(I)\right) .
$$

The equation

$$
\tau[y]-\lambda w y=0 \quad(\lambda \in \mathbb{C}) \text { on } I
$$

is said to be regular at the left end point $a \in \mathbb{R}$, if for all $X \in(a, b), a \in \mathbb{R}$; $w, p_{r s} \in L^{1}[a, X],(r, s=1, \ldots, n)$. Otherwise (2.10) is said to be singular at $a$. If (2.10) is regular at both end points, then it is said to be regular; in this case we have,

$$
a, b \in \mathbb{R}, \quad w, p_{r s} \in L^{1}(a, b), \quad(r, s=1, \ldots, n) .
$$

We will be concerned with the case when $a$ is a regular end point of (2.10), the end point $b$ being allowed to be either regular or singular. Note that, in view of (2.8), an end point of $I$ is regular for (2.10), if and only if it is regular for the equation

$$
\tau^{+}[z]-\bar{\lambda} w z=0 \quad(\lambda \in \mathbb{C}) \text { on } I .
$$

Note that, at a regular end point $a, y^{[r-1]}(a)\left(z_{+}^{[r-1]}(a)\right), r=1, \ldots, n$, is defined for all $u \in V(\tau)\left(v \in V\left(\tau^{+}\right)\right)$. Set

$$
\begin{aligned}
D(\tau) & :=\left\{y: y \in V(\tau), y, w^{-1} \tau[y] \in L_{w}^{2}(a, b)\right\}, \\
D\left(\tau^{+}\right) & :=\left\{z: z \in V\left(\tau^{+}\right), z, w^{-1} \tau^{+}[z] \in L_{w}^{2}(a, b)\right\} .
\end{aligned}
$$

The subspaces $D(\tau)$ and $D\left(\tau^{+}\right)$of $L_{w}^{2}(a, b)$ are domains of the so-called maximal operators $T(\tau)$ and $T\left(\tau^{+}\right)$, respectively, defined by

$$
\begin{gathered}
T(\tau) y:=w^{-1} \tau[y] \quad(y \in D(\tau)), \\
T\left(\tau^{+}\right) z:=w^{-1} \tau^{+}[z], \quad\left(z \in D\left(\tau^{+}\right)\right) .
\end{gathered}
$$


For the regular problem, the minimal operators $T_{0}(\tau)$ and $T_{0}\left(\tau^{+}\right)$are the restrictions of $w^{-1} \tau[y]$ and $w^{-1} \tau^{+}[z]$ to the subspaces

$$
\begin{aligned}
D_{0}(\tau) & :=\left\{y: y \in D(\tau), y^{[r-1]}(a)=y^{[r-1]}(b)=0, r=1, \ldots, n\right\}, \\
D_{0}\left(\tau^{+}\right) & :=\left\{z: z \in D\left(\tau^{+}\right), z_{+}^{[r-1]}(a)=z_{+}^{[r-1]}(b)=0, r=1, \ldots, n\right\},
\end{aligned}
$$

respectively. The subspaces $D_{0}(\tau)$ and $D_{0}\left(\tau^{+}\right)$are dense in $L_{w}^{2}(a, b)$, and $T_{0}(\tau)$ and $T_{0}\left(\tau^{+}\right)$are closed operators (see [2, 3, 6] and [13, Section 3]).

In the singular problem, we first introduce the operators $T_{0}^{\prime}(\tau)$ and $T_{0}^{\prime}\left(\tau^{+}\right)$; $T_{0}^{\prime}(\tau)$ being the restriction of $w^{-1} \tau[\cdot]$ to the subspace

$$
D_{0}^{\prime}(\tau):=\{y: y \in D(\tau), \operatorname{supp} y \subset(a, b)\}
$$

and with $T_{0}^{\prime}\left(\tau^{+}\right)$defined similarly. These operators are densely defined and closable in $L_{w}^{2}(a, b)$, and we defined the minimal operators $T_{0}(\tau), T_{0}\left(\tau^{+}\right)$to be their respective closures (see [2] and [13, Section 5]). We denote the domains of $T_{0}(\tau)$ and $T_{0}\left(\tau^{+}\right)$by $D_{0}(\tau)$ and $D_{0}\left(\tau^{+}\right)$, respectively. It can be shown that

$$
\begin{gathered}
y \in D_{0}(\tau) \Longrightarrow y^{[r-1]}(a)=0 \quad(r=1, \ldots, n), \\
z \in D_{0}\left(\tau^{+}\right) \Longrightarrow z_{+}^{[r-1]}(a)=0 \quad(r=1, \ldots, n),
\end{gathered}
$$

because we are assuming that $a$ is a regular end point. Moreover, in both regular and singular problems, we have

$$
T_{0}^{*}(\tau)=T\left(\tau^{+}\right), \quad T^{*}(\tau)=T_{0}\left(\tau^{+}\right),
$$

see [13, Section 5] in the case when $\tau=\tau^{+}$and compare it with treatment in [2, Section III.10.3] and [3] in general case.

3. Some technical lemmas. The proof of the general theorem is based on the results in this section. We start by listing some properties and results of quasi-differential expressions $\tau_{1}, \tau_{2}, \ldots, \tau_{n}$ each of order $n$. For proofs, the reader is referred to $[4,12,13,14]$.

$$
\begin{gathered}
\left(\tau_{1}+\tau_{2}\right)^{+}=\tau_{1}^{+}+\tau_{2}^{+}, \\
\left(\tau_{1} \tau_{2}\right)^{+}=\tau_{2}^{+} \tau_{1}^{+}, \quad(\lambda \tau)^{+}=\bar{\lambda} \tau^{+}, \quad \text { for } \lambda \text { is a complex number. }
\end{gathered}
$$

A consequence of properties (3.1) is that if $\tau^{+}=\tau$, then $P(\tau)^{+}=P\left(\tau^{+}\right)$for $P$ is any polynomial with complex coefficients. Also we note that the leading coefficients of a product is the product of the leading coefficients. Hence the product of regular differential expressions is regular. 
LEMmA 3.1 (cf. [4, Theorem 1]). Suppose that $\tau_{j}$ is a regular differential expression on the interval $[0, b]$ such that the minimal operator $T_{0}\left(\tau_{j}\right)$ has property $(C)$ for $j=1,2, \ldots, n$. Then

(i) the product operator $\prod_{j=1}^{n}\left[T_{0}\left(\tau_{j}\right)\right]$ is closed and has dense domain, prop$\operatorname{erty}(C)$, and

$$
\operatorname{def}\left[\prod_{j=1}^{n} T_{0}\left(\boldsymbol{\tau}_{j}\right)\right]=\sum_{j=1}^{n} \operatorname{def}\left[T_{0}\left(\boldsymbol{\tau}_{j}\right)\right] ;
$$

(ii) the operators $T_{0}\left(\tau_{1} \tau_{2} \cdots \tau_{n}\right)$ and $\prod_{j=1}^{n}\left[T_{0}\left(\tau_{j}\right)\right]$ are not equal in general, that is, $\left[T_{0}\left(\tau_{1} \tau_{2} \cdots \tau_{n}\right)\right] \subseteq \prod_{j=1}^{n}\left[T_{0}\left(\tau_{j}\right)\right]$.

LEMMA 3.2 (cf. [4, Theorem 2]). Let $\tau_{1}, \tau_{2}, \ldots, \tau_{n}$ be regular differential expressions on $[0, b]$. Suppose that $T_{0}\left(\tau_{j}\right)$ satisfies property $(C)$ for $j=1,2, \ldots, n$. Then

$$
T_{0}\left(\boldsymbol{\tau}_{1} \boldsymbol{\tau}_{2} \cdots \boldsymbol{\tau}_{n}\right)=\prod_{j=1}^{n} T_{0}\left(\boldsymbol{\tau}_{j}\right)
$$

if and only if the following partial separation condition is satisfied:

$$
\left\{f \in L_{w}^{2}(a, b), f^{[s-1]} \in A C_{\mathrm{loc}}[a, b)\right\},
$$

where $s$ is the order of product expression $\left(\tau_{1} \tau_{2} \cdots \tau_{n}\right)$ and $\left(\tau_{1} \tau_{2} \cdots \tau_{n}\right)^{+} f \in$ $L_{w}^{2}(a, b)$ together imply that $\left(\prod_{j=1}^{k}\left(\tau_{j}^{+}\right)\right) f \in L_{w}^{2}(a, b), k=1, \ldots, n-1$.

Furthermore, $T_{0}\left(\tau_{1} \tau_{2} \cdots \tau_{n}\right)=\prod_{j=1}^{n} T_{0}\left(\tau_{j}\right)$ if and only if

$$
\operatorname{def}\left[T_{0}\left(\tau_{1} \tau_{2} \cdots \tau_{n}\right)\right]=\sum_{j=1}^{n} \operatorname{def}\left[T_{0}\left(\tau_{j}\right)\right]
$$

then the product $\left(\tau_{1} \tau_{2} \cdots \tau_{n}\right)$ is partially separated expressions in $L_{w}^{2}(0, b)$ whenever property (3.4) holds.

LEMMA 3.3 (cf. [4, Corollary 1]). Let $\tau_{j}$ be a regular differential expression on $[0, b]$ for $j=1, \ldots, n$. If all solutions of the differential equations $\left(\tau_{j}\right) y=0$ and $\left(\tau_{j}^{+}\right) z=0$ on $[0, b]$ are in $L_{w}^{2}(0, b)$ for $j=1, \ldots, n$, then all solutions of $\left(\tau_{1} \tau_{2} \cdots \tau_{n}\right) y=0$ and $\left(\tau_{1} \tau_{2} \cdots \tau_{n}\right)^{+} z=0$ are in $L_{w}^{2}(0, b)$.

The special case of Lemma 3.3 when $\tau_{j}=\tau$ for $j=1,2, \ldots, n$ and $\tau$ is symmetric was established in [14]. In this case, it is easy to see that the converse also holds. If all solutions of $\tau^{n} u=0$ are in $L_{w}^{2}(0, b)$, then all solutions of $\tau y=0$ must be in $L_{w}^{2}(0, b)$. In general, if all solutions of $\left(\tau_{1} \tau_{2} \cdots \tau_{n}\right) y=0$ 
are in $L_{w}^{2}(0, b)$, then all solutions of $\tau_{n} y=0$ are in $L_{w}^{2}(0, b)$ since these also the solutions of $\left(\tau_{1} \tau_{2} \cdots \tau_{n}\right) y=0$. If all solutions of the adjoints equation $\left(\tau_{1} \tau_{2} \cdots \tau_{n}\right)^{+} z=0$ are also in $L_{w}^{2}(0, b)$, then it follows similarly that all solutions of $\tau_{1}^{+} z=0$ are in $L_{w}^{2}(0, b)$. So, in particular, for $n=2$ we have established the following lemma.

LEMMA 3.4. Suppose that $\tau_{1}, \tau_{2}$, and $\tau_{1} \tau_{2}$ are all regular expressions on $[0, b]$. Then the product is in the maximal deficiency case at $b$ if and only if both $\tau_{1}, \tau_{2}$ are in the maximal deficiency case at $b$, see [4, Corollary 2] for more details.

Denote by $S(\tau)$ and $S\left(\tau^{+}\right)$the sets of all solutions of the equations

$$
\left(\prod_{j=1}^{n} \tau_{j}\right) y=0, \quad\left(\prod_{j=1}^{n} \tau_{j}^{+}\right) z=0,
$$

respectively. Let $\phi_{k}(t), k=1,2, \ldots, n^{2}$, denote the solutions of the homogeneous equation $\left(\prod_{j=1}^{n} \tau_{j}\right) y=0$ determined by the initial conditions

$$
\phi_{k}^{[r]}\left(t_{0}\right)=\delta_{k, r+1} \quad \forall t_{0} \in[0, b]
$$

(where $k=1,2, \ldots, n^{2} ; r=0,1, \ldots, n^{2}-1$ ). Let $\phi_{k}^{+}(t), k=1,2, \ldots, n^{2}$, denote the solutions of the homogeneous equation $\left(\prod_{j=1}^{n} \tau_{j}^{+}\right) z=0$ determined by the initial conditions

$$
\left(\phi_{k}^{+}\right)^{[r]}\left(t_{0}\right)=(-1)^{k+r} \delta_{k, n^{2}-r} \quad \forall t_{0} \in[0, b],
$$

where $k=1,2, \ldots, n^{2} ; r=0,1, \ldots, n^{2}-1$.

REMARK 3.5. If all solutions $\phi_{k}(t), \phi_{k}^{+}(t), k=1,2, \ldots, n^{2}$, of $\left(\prod_{j=1}^{n} \tau_{j}\right) y=0$ and $\left(\prod_{j=1}^{n} \tau_{j}^{+}\right) z=0$, respectively are bounded $\left(L_{w}^{2}\right.$-bounded) on $[0, b)$, then $S(\tau)$ and $S\left(\tau^{+}\right)$are bounded ( $L^{2}$-bounded) and hence $S(\tau) \cup S\left(\tau^{+}\right)$is bounded ( $L^{2}$-bounded) on [0,b); see [6] and [7, Lemmas 3.4 and 3.5].

The next lemma is a form of the variation of parameters formula of a general quasidifferential equation, see [6, Section 3] and [7, 13].

LEMMA 3.6. For $f$ locally integrable, the solution $\phi$ of the quasidifferential equation

$$
\left(\prod_{j=1}^{n} \tau_{j}\right) y=w f \quad \text { on }[0, b)
$$


satisfying

$$
\phi^{[r]}\left(t_{0}\right)=\alpha_{r+1} \quad \forall t_{0} \in[0, b), r=0,1, \ldots, n^{2}-1
$$

is given by

$$
\phi(t)=\sum_{j=1}^{n^{2}} \alpha_{j} \phi_{j}(t)+\frac{1}{i^{n^{2}}} \sum_{j, k=1}^{n^{2}} \zeta^{j k} \phi_{j}(t) \int_{t_{0}}^{t} \overline{\phi_{k}^{+}(s)} f(s) w(s) d s
$$

for some $\alpha_{1}, \alpha_{2}, \ldots, \alpha_{n^{2}} \in \mathbb{C}$, where $\phi_{j}(t)$ and $\phi_{k}^{+}(t), j, k=1,2, \ldots, n^{2}$, are solutions of the equations in (3.6), respectively, $\zeta^{j k}$ is a constant which is independent of $t$.

In the sequel, we will require the following nonlinear integral inequality which generalizes those integral inequalities used in $[1,5,9,10]$.

LEMMA 3.7 (cf. [5, 10]). Let $u(t), v(t), f(t, s), g_{i}(t, s)$, and $h_{i}(t, s) \quad(i=$ $\left.1,2, \ldots, n^{2}\right)$ be nonnegative continuous functions defined on the interval I and $I \times I$, respectively, here $I=(0, c), 0<c \leq \infty$, with their ranges in $\mathbb{R}^{+}$. Let $v(t)$ be nondecreasing on $I$, and $f(t, s), g_{i}(t, s)$, and $h_{i}(t, s),\left(i=1,2, \ldots, n^{2}\right)$ be nondecreasing in $t$ for each $s \in I$ fixed. Suppose that the inequality

$$
u(t) \leq v(t)+\int_{0}^{t} f(t, s) u(s) d s+\sum_{j=1}^{n^{2}} \int_{0}^{t} g_{i}(t, s)\left[\int_{0}^{s} h_{i}(t, s)[u(\tau)]^{\sigma} d \tau\right] d s
$$

holds for all $t \in I$, where $\sigma \in(0,1]$ is constant. Then

(i) if $0<\sigma<1$,

$$
u(t) \leq\left[[v(t) F(t)]^{1-\sigma}+(1-\sigma) \sum_{i=1}^{n^{2}} G_{i}(t) F(t) \int_{0}^{t} h_{i}(t, s) d s\right]^{1 /(1-\sigma)}, \quad t \in I,
$$

(ii) if $\sigma=1$,

$$
u(t) \leq v(t) \exp \int_{0}^{t}\left[f(t, s)+\sum_{i=1}^{n^{2}} G_{i}(t) F(t) h_{i}(t, s)\right] d s,
$$

where

$$
F(t)=\exp \int_{0}^{t} f(t, s) d s, \quad G_{i}(t)=\int_{0}^{t} g_{i}(s) d s, \quad i=1,2, \ldots, n^{2} .
$$

COROLlary 3.8 (cf. $[9,10])$. Let $u(t), f_{1}(t), f_{2}(t), g_{1}(t, s)$, and $g_{2}(t, s)$ be nonnegative continuous functions defined on the intervals $I=[0, b)$ and $I \times I$, 
respectively. Suppose that the inequality

$$
\begin{aligned}
u(t) \leq & C+\int_{0}^{t} f_{1}(s) u(s) d s+\int_{0}^{t} f_{2}(s) u^{\sigma}(s) d s \\
& +\int_{0}^{t}\left(\int_{0}^{s} g_{1}(s, x) u(x) d x\right)+\int_{0}^{t}\left(g_{2}(s, x) u^{\sigma}(x)\right) d s
\end{aligned}
$$

holds for all $t \in[0, b)$, where $\sigma \in(0,1]$ and $C$ is constant. Then

$$
\begin{aligned}
u(t) \leq & {\left[C^{(1-p)}+(1-\sigma)\right.} \\
& \times \int_{0}^{t}\left[f_{2}(s)+\int_{0}^{s} g_{2}(s, x) d x\right] \\
& \left.\quad \times \exp \left[(1-\sigma) \int_{0}^{s}\left[f_{1}(\tau)+\int_{0}^{s} g_{1}(\tau, x) d x\right] d \tau\right] d s\right]^{1 /(1-p)} \\
& \times \exp \left(\int_{0}^{t}\left[f_{1}(s)+\int_{0}^{s} g_{1}(s, x) d x\right] d s\right) .
\end{aligned}
$$

4. Boundedness of solutions. In this section, we consider the question of determining conditions under which all solutions of (1.2) are bounded and $L_{w}^{2}$-bounded.

Suppose there exist nonnegative continuous functions $e_{1}(t), e_{2}(t), e_{3}(t)$, $r_{1}(t), r_{2}(t), K_{0}(t, s)$, and $K_{i}(t-s)$ on $[0, b), 0<b \leq \infty ; i=1,2, \ldots, n^{2}-1$ such that,

$$
\begin{aligned}
& \left|F\left(t, y, \int_{0}^{t} g\left(t, s, y, y^{\prime}, \ldots, y^{\left(n^{2}-1\right)}(s)\right) d s\right)\right| \\
& \leq e_{1}(t)+r_{1}(t)|y(t)|^{\sigma}+r_{2}(t)\left[\int_{0}^{t}\left[e_{2}(t)+e_{3}(s)+K_{0}(t, s)|y(s)|^{\sigma}\right] d s\right. \\
& \left.+\left|\int_{0}^{t} \sum_{i=1}^{n^{2}-1} K_{i}(t-s) y^{(i)}(s) d s\right|\right],
\end{aligned}
$$

for $t, s \geq 0$ and some $\sigma \in[0,1]$; see $[5,9,10]$.

THEOREM 4.1. Suppose that (4.1) is satisfied with $\sigma=1, S(\tau) \cup S\left(\tau^{+}\right)$is bounded on $[0, b)$, and that
(a) $k_{i}^{(\ell)}(0)=0$ for all $\ell=0,1, \ldots, i-1 ; i=1,2, \ldots, n^{2}-1$,
(b) $e_{1}(t), r_{1}(t)$, and $r_{2} k_{i}^{(\ell)}(t) \in L_{w}^{1}(0, b), \ell=0,1, \ldots, i-1 ; i=1,2, \ldots, n^{2}-1$,
(c) the following integrals are bounded at $t \rightarrow b$, 

(i) $\int_{0}^{t} r_{2}(s)\left(\int_{0}^{s}\left[e_{2}(x)+e_{3}(x)\right] d x\right) w(s) d s$,
(ii) $\int_{0}^{t} r_{2}(s)\left(\int_{0}^{s} K_{0}(s, x) d x\right) w(s) d s$,
(iii) $\int_{0}^{t} r_{2}(s)\left(\sum_{i=1}^{n^{2}-1} \int_{0}^{s}\left|\left(\partial^{i} / \partial x^{i}\right) K_{i}(s-x)\right| d x\right) w(s) d s$.

Then all solutions of (1.2) are also bounded on $[0, b)$.

Proof. Note that (4.1) implies that all solutions are defined on $[0, b)$. Let $\left\{\phi_{1}(t), \ldots, \phi_{n^{2}}(t)\right\}$ and $\left\{\phi_{1}^{+}(t), \ldots, \phi_{n^{2}}^{+}(t)\right\}$ be two sets of linearly independent solutions of the equations in (3.6), respectively, and let $\phi(t)$ be any solution of $(1.2)$ on $[0, b)$, then by Lemma 3.6, we have

$$
\phi(t)=\sum_{j=1}^{n^{2}} \alpha_{j} \phi_{j}(t)+\frac{1}{i^{n^{2}}} \sum_{j, k=1}^{n^{2}} \zeta^{j k} \phi_{j}(t) \int_{0}^{t} \overline{\phi_{k}^{+}(s)} F(s) w(s) d s .
$$

Hence,

$$
\begin{aligned}
|\phi(t)| \leq & \sum_{j=1}^{n^{2}}\left|\alpha_{j}\right|\left|\phi_{j}(t)\right| \\
+ & \sum_{j, k=1}^{n^{2}}\left|\zeta^{j k}\right|\left|\phi_{j}(t)\right| \\
& \times \int_{0}^{t}\left|\overline{\phi_{k}^{+}(s)}\right|\left[e_{1}(s)+r_{1}(s)|\phi(s)|\right. \\
& +r_{2}(s)\left[\int_{0}^{s}\left[e_{2}(s)+e_{3}(x)+K_{0}(s, x)|\phi(x)|\right] d x\right. \\
& \left.\left.+\left|\int_{0}^{s} \sum_{i=1}^{n^{2}-1} K_{i}(s-x) \phi^{(i)}(x) d x\right|\right]\right] w(s) d s .
\end{aligned}
$$

Since $\phi_{k}^{+}(t)$ is bounded on $[0, b), k=1, \ldots, n^{2}$, and $e_{1}(t) \in L_{w}^{1}(0, b)$, then $\phi_{k}^{+}(t) e_{1}(t) \in L_{w}^{1}(0, b), k=1,2, \ldots, n^{2}$. Setting

$$
C_{k}=\int_{0}^{t}\left|\overline{\phi_{k}^{+}(s)}\right| e_{1}(s) w(s) d s,
$$

and integrating the last integral in (4.3) by parts, we have

$$
\begin{aligned}
& \sum_{i=1}^{n^{2}-1} \int_{0}^{s} K_{i}(s-x) \phi^{(i)}(x) d x \\
& \quad=\sum_{\ell=0}^{i-1}(-1)^{\ell+1} K_{i}^{(\ell)}(s) \phi^{(i-1-\ell)}(0)+(-1)^{i} \int_{0}^{s} \frac{\partial^{i}}{\partial x^{i}} K_{i}(s-x) \phi(x) d x,
\end{aligned}
$$



where $K_{i}^{(\ell)}(0)=0$ for all $\ell=0,1, \ldots, i-1 ; i=1,2, \ldots, n^{2}-1$. Then (4.3) becomes

$$
\begin{aligned}
|\phi(t)| \leq & \sum_{j=1}^{n^{2}}\left(C_{j}+\left|\alpha_{j}\right|\right)\left|\phi_{j}(t)\right| \\
+ & \sum_{j, k=1}^{n^{2}}\left|\zeta^{j k}\right|\left|\phi_{j}(t)\right| \\
& \times \int_{0}^{t}\left|\overline{\phi_{k}^{+}(s)}\right|\left[r_{1}(s)|\phi(s)|\right. \\
& +r_{2}(s)\left(\int_{0}^{s}\left[e_{2}(s)+e_{3}(x)+K_{0}(s, x)|\phi(x)|\right] d x\right. \\
& +\sum_{i=1}^{n^{2}-1} \sum_{\ell=0}^{i-1}\left|K_{i}^{(\ell)}(s)\right|\left|\phi^{(i-1-\ell)}(0)\right| \\
& \left.\left.+\sum_{i=1}^{n^{2}-1} \int_{0}^{s}\left|\frac{\partial^{i}}{\partial x^{i}} K_{i}(s-x)\right||\phi(x)| d x\right)\right] w(s) d s,
\end{aligned}
$$

where $\left|\phi^{(i-1-\ell)}(0)\right| \leq \beta$ for all $\ell=0,1, \ldots, i-1 ; i=0, \ldots, n^{2}-1$. Let

$$
\begin{aligned}
& h(t)= \sum_{j=1}^{n^{2}}\left(C_{j}+\left|\alpha_{j}\right|\right)\left|\phi_{j}(t)\right| \\
&+\sum_{j, k=1}^{n^{2}}\left|\zeta^{j k}\right|\left|\phi_{j}(t)\right| \int_{0}^{t}\left|\overline{\phi_{k}^{+}(s)}\right|\left[r _ { 2 } ( s ) \left[\int_{0}^{s}\left[e_{2}(s)+e_{3}(x)\right] d x\right.\right. \\
&\left.\left.+\sum_{i=1}^{n^{2}-1} \sum_{\ell=0}^{i-1}\left|K_{i}^{(\ell)}(s)\right| \beta\right]\right] w(s) d s .
\end{aligned}
$$

Then (4.6) becomes

$$
\begin{aligned}
|\phi(t)| \leq & h(t) \\
+ & \sum_{j, k=1}^{n^{2}}\left|\zeta^{j k}\right|\left|\phi_{j}(t)\right| \\
\times \int_{0}^{t}\left|\overline{\phi_{k}^{+}(s)}\right| & {\left[r_{1}(s)|\phi(s)|\right.} \\
& +r_{2}(s)\left[\int_{0}^{s} K_{0}(s, x)|\phi(x)| d x\right. \\
& \left.\left.+\sum_{i=1}^{n^{2}-1} \int_{0}^{s}\left|\frac{\partial^{i}}{\partial x^{i}} K_{i}(s-x)\right||\phi(x)| d x\right]\right] w(s) d s .
\end{aligned}
$$


From our assumptions and conditions (i) and (ii), it follows that $h(t)$ is bounded on $[0, b)$. Applying Lemma 3.7 with $\sigma=1$, we obtain

$$
\begin{aligned}
&|\phi(t)| \\
& \leq h(t) \exp \left\{\sum_{j, k=1}^{n^{2}}\left|\zeta^{j k}\right|\left|\phi_{j}(t)\right|\right. \\
& \times \int_{0}^{t}\left|\overline{\phi_{k}^{+}(s)}\right|\left[r_{1}(s)+r_{2}(s)\left[\int _ { 0 } ^ { s } \left[K_{0}(s, x)\right.\right.\right. \\
&\left.\left.\left.\left.+\sum_{i=1}^{n^{2}-1}\left|\frac{\partial^{i}}{\partial x^{i}} K_{i}(s-x)\right|\right] d x\right]\right] w(s) d s\right\},
\end{aligned}
$$

and hence our assumptions and conditions (i), (ii), and (iii) yield that $\phi(t)$ is bounded on $[0, b)$.

THEOREM 4.2. Suppose that $S(\tau) \cup S\left(\tau^{+}\right) \subset L_{w}^{2}(0, b)$ with $\sigma=1$, and that

(i) $r_{1}(t)$ and $r_{2}(t)$ are bounded on $[0, b)$,

(ii) $e_{1}(s)$ and $K_{i}^{(\ell)}(s) \in L_{w}^{2}(0, b)$ for all $\ell=0,1, \ldots, i-1 ; i=1,2, \ldots, n^{2}-1$,

(iii) $\int_{0}^{t}\left[\int_{0}^{s}\left[e_{2}(s)+e_{3}(x)\right] d x\right]^{2} w(s) d s<\infty$,

(iv) $\int_{0}^{t}\left[\int_{0}^{s}\left[(1 / w)\left(K_{0}^{2}(s, x)\right)+\left[\sum_{i=1}^{n^{2}-1}\left|\left(\partial^{i} / \partial x^{i}\right) K_{i}(s-x)\right|\right]^{2}\right] d x\right] w(s) d s<\infty$. Then all solutions of (1.2) are in $L_{w}^{2}(0, b)$.

Proof. The proof is the same up to (4.5), since $\phi_{k}^{+}(s), e_{1}(s) \in L_{w}^{2}(0, b)$ (see Lemma 3.3), then $\phi_{k}^{+}(s) e_{1}(s) \in L_{w}^{1}(0, b), k=1,2, \ldots, n^{2}$, for all $s \in(0, b)$. By using (4.4) and applying the Cauchy-Schwartz inequality to the integral in (4.6), we have

$$
\begin{aligned}
&|\phi(t)| \leq \sum_{j=1}^{n^{2}}\left(C_{j}+\left|\alpha_{j}\right|\right)\left|\phi_{j}(t)\right| \\
&+ \sum_{j, k=1}^{n^{2}}\left|\zeta^{j k}\right|\left|\phi_{j}(t)\right| \\
& \times \int_{0}^{t}\left|\overline{\phi_{k}^{+}(s)}\right|\left[\left(\int_{0}^{t}\left|\overline{\phi_{k}^{+}(s)}\right|^{2} r_{1}^{2}(s) w(s) d s\right)^{1 / 2}\left(\int_{0}^{t}|\phi(s)|^{2} w(s) d s\right)^{1 / 2}\right. \\
&+\left(\int_{0}^{t}\left|\overline{\phi_{k}^{+}(s)}\right|^{2} r_{2}^{2}(s) w(s) d s\right)^{1 / 2} \\
& \times \\
& \times\left\{\left(\int_{0}^{t}\left[\int_{0}^{s}\left[e_{2}(s)+e_{3}(x)\right] d x\right]^{2} w(s) d s\right)^{1 / 2}\right. \\
&+\left(\int_{0}^{t}\left[\int_{0}^{s} K_{0}(s, x)|\phi(x)| d x\right]^{2} w(s) d s\right)^{1 / 2}
\end{aligned}
$$




$$
\begin{aligned}
& +\left(\int_{0}^{t}\left[\sum_{i=1}^{n^{2}-1} \sum_{\ell=0}^{i-1}\left|K_{i}^{[\ell]}(s) \beta\right|\right]^{2} w(s) d s\right)^{1 / 2} \\
& \left.\left.+\left(\int_{0}^{t}\left[\sum_{i=1}^{n^{2}-1}\left|\frac{\partial^{i}}{\partial x^{i}} K_{i}(s-x) \phi(x)\right| d x\right]^{2} w(s) d s\right)^{1 / 2}\right\}\right] .
\end{aligned}
$$

Since $r_{1}(s), r_{2}(s)$ are bounded on $[0, b)$ and $\phi_{k}^{+}(s) \in L_{w}^{2}(0, b)$, then $\phi_{k}^{+}(s) r_{1}(s)$, $\phi_{k}^{+}(s) r_{2}(s) \in L_{w}^{2}(0, b) ; k=1,2, \ldots, n^{2}$ for all $s \in[0, b)$ and hence there exist positive constants $\zeta_{1}, \zeta_{2}$ such that

$$
\left\|\phi_{k}^{+}(s) r_{i}(s)\right\|_{L_{w}^{2}(0, b)} \leq \xi_{i} \quad \forall k=1,2, \ldots, n^{2} ; i=1,2 .
$$

Therefore (4.10) becomes

$$
\begin{aligned}
& |\phi(t)| \\
& \leq \sum_{j=1}^{n^{2}}\left(C_{j}+\left|\alpha_{j}\right|\right)\left|\phi_{j}(t)\right| \\
& +\sum_{j, k=1}^{n^{2}}\left|\zeta^{j k}\right|\left|\phi_{j}(t)\right|\left[\xi_{1}\left(\int_{0}^{t}|\phi(s)|^{2} w(s) d s\right)^{1 / 2}\right. \\
& +\xi_{2}\left\{\left(\int_{0}^{t}\left[\int_{0}^{s}\left[e_{2}(s)+e_{3}(x)\right] d x\right]^{2} w(s) d s\right)^{1 / 2}\right. \\
& +\left(\int_{0}^{t}\left[\int_{0}^{s} K_{0}(s, x)|\phi(x)| d x\right]^{2} w(s) d s\right)^{1 / 2} \\
& +\left(\int_{0}^{t}\left[\sum_{i=1}^{n^{2}-1} \sum_{\ell=0}^{i-1}\left|K_{i}^{(\ell)}(s) \beta\right|\right]^{2} w(s) d s\right)^{1 / 2} \\
& \left.\left.+\left(\int_{0}^{t}\left[\sum_{i=1}^{n^{2}-1}\left|\frac{\partial^{i}}{\partial x^{i}} K_{i}(s-x) \phi(x)\right| d x\right]^{2} w(s) d s\right)^{1 / 2}\right\}\right] .
\end{aligned}
$$

Let

$$
\begin{aligned}
h(t)=\sum_{j=1}^{n^{2}}\left(C_{j}+\left|\alpha_{j}\right|\right)\left|\phi_{j}(t)\right| & \\
+\sum_{j, k=1}^{n^{2}}\left|\zeta^{j k}\right|\left|\phi_{j}(t)\right| & {\left[\xi_{2}\left(\int_{0}^{t}\left[\int_{0}^{s}\left[e_{2}(s)+e_{3}(x)\right] d x\right]^{2} w(s) d s\right)^{1 / 2}\right.} \\
& \left.+\left(\int_{0}^{t}\left[\sum_{i=1}^{n^{2}-1} \sum_{\ell=0}^{i-1}\left|K_{i}^{(\ell)}(s) \beta\right|\right]^{2} w(s) d s\right)^{1 / 2}\right],
\end{aligned}
$$


then

$$
\begin{aligned}
|\phi(t)| \leq & h(t)+\sum_{j, k=1}^{n^{2}}\left|\zeta^{j k}\right|\left|\phi_{j}(t)\right| \\
\times & {\left[\xi_{1}\left(\int_{0}^{t}|\phi(s)|^{2} w(s) d s\right)^{1 / 2}\right.} \\
& +\xi_{2}\left\{\left(\int_{0}^{t}\left[\int_{0}^{s} K_{0}(s, x)|\phi(x)| d x\right]^{2} w(s) d s\right)^{1 / 2}\right. \\
& \left.\left.+\left(\int_{0}^{t}\left[\sum_{i=1}^{n^{2}-1}\left|\frac{\partial^{i}}{\partial x^{i}} K_{i}(s-x) \phi(x)\right| d x\right]^{2} w(s) d s\right)^{1 / 2}\right\}\right] .
\end{aligned}
$$

Applying the Cauchy-Schwartz inequality and squaring both sides of (4.1), we have

$$
\begin{aligned}
|\phi(t)|^{2} \leq & 2 h^{2}(t) \\
& +4 \sum_{j, k=1}^{n^{2}}\left|\zeta^{j k}\right|\left|\phi_{j}(t)\right|^{2} \\
\times & {\left[\xi_{1}^{2}\left(\int_{0}^{t}|\phi(s)|^{2} w(s) d s\right)\right.} \\
& +\xi_{2}^{2} \int_{0}^{t}\left(\int_{0}^{s} \frac{1}{w}\left(K_{0}^{2}(s, x)+\left[\sum_{i=1}^{n^{2}-1}\left|\frac{\partial^{i}}{\partial x^{i}} K_{i}(s-x)\right| d x\right]^{2}\right) d x\right) \\
& \left.\times\left(\int_{0}^{t}|\phi(s)|^{2} w(s) d s\right)\right] .
\end{aligned}
$$

If $u(t)=\int_{0}^{t}|\phi(s)|^{2} w(s) d s$, then

$$
\begin{aligned}
& u(t) \leq 2 \int_{0}^{t} h^{2}(s) w(s) d s \\
&+4 \xi_{1}^{2} \sum_{j, k=1}^{n^{2}}\left|\zeta^{j k}\right| \int_{0}^{t}\left|\phi_{j}(s)\right|^{2} w(s) d s \\
&+4 \xi_{2}^{2} \sum_{j, k=1}^{n^{2}} \zeta^{j k} \int_{0}^{t}\left|\phi_{j}(s)\right|^{2} \\
& \times\left[\int_{0}^{s}\left(\int_{0}^{\tau} \frac{1}{w}\left(K_{0}^{2}(s, x)+\left[\sum_{i=1}^{n^{2}-1}\left|\frac{\partial^{i}}{\partial x^{i}} K_{i}(s-x)\right|\right]^{2}\right) d x\right)\right. \\
&\times u(\tau) w(\tau) d \tau] w(s) d s .
\end{aligned}
$$


From conditions (ii) and (iii), it follows that the integral $\int_{0}^{t} h^{2}(s) w(s) d s$ will be finite and by using Lemma 3.7, we obtain

$$
\begin{aligned}
& u(t) \leq\left(2 \int_{0}^{t} h^{2}(s) w(s) d s\right) \\
& \times \exp \left\{4 \xi_{1}^{2} \sum_{j, k=1}^{n^{2}}\left|\zeta^{j k}\right| \int_{0}^{t}\left|\phi_{j}(s)\right|^{2} w(s) d s\right. \\
&+4 \xi_{1}^{2} \sum_{j, k=1}^{n^{2}} \zeta^{j k} \int_{0}^{t}\left|\phi_{j}(s)\right|^{2} \\
& \times\left[\int_{0}^{s}\left(\int_{0}^{\tau} \frac{1}{w}\left(K_{0}^{2}(s, x)+\left[\sum_{i=1}^{n^{2}-1}\left|\frac{\partial^{i}}{\partial x^{i}} K_{i}(s-x)\right|\right]^{2}\right) d x\right)\right. \\
&\times w(\tau) d \tau] w(s) d s\} .
\end{aligned}
$$

Hence our assumption and condition (iv) yield that $\phi(t) \in L_{w}^{2}(0, b)$.

Next, we consider (4.1) with $0 \leq \sigma<1$, and we have the following results.

THEOREM 4.3. Suppose that $S(\tau) \cup S\left(\tau^{+}\right)$is bounded on $[0, b)$ and that

(i) $e_{1}(s)$ and $r_{1}(s) \in L_{w}^{2}(0, b)$ for all $s \in[0, b)$,

(ii) $\int_{0}^{t} r_{2}(s)\left(\int_{0}^{s}\left[e_{2}(s)+e_{3}(x)\right] d x\right) w(s) d s<\infty$,

(iii) $\int_{0}^{t} r_{2}(s)\left(\sum_{i=1}^{n^{2}-1} \sum_{\ell=0}^{i-1}\left|K_{i}^{(\ell)}(s)\right|\right) w(s) d s<\infty$,

(iv) $\int_{0}^{t} r_{2}(s)\left(\int_{0}^{s} K_{0}(s, x) d x\right) w(s) d s<\infty$,

(v) $\int_{0}^{t} r_{2}(s)\left(\sum_{i=1}^{n^{2}-1} \int_{0}^{s}\left|\left(\partial^{i} / \partial x^{i}\right) K_{i}(s-x)\right| d x\right) w(s) d s<\infty$.

Then all solutions of (1.2) are bounded in $[0, b)$.

Proof. For $0 \leq \sigma<1$, the proof is the same up to (4.6). In this case (4.6) becomes

$$
\begin{aligned}
|\phi(t)| \leq & \sum_{j=1}^{n^{2}}\left(C_{j}+\left|\alpha_{j}\right|\right)\left|\phi_{j}(t)\right| \\
+ & \sum_{j, k=1}^{n^{2}}\left|\zeta^{j k}\right|\left|\phi_{j}(t)\right| \\
& \quad \times \int_{0}^{t}\left|\overline{\phi_{k}^{+}(s)}\right|\left[r_{1}(s)|\phi(s)|^{\sigma}\right.
\end{aligned}
$$




$$
\begin{aligned}
+r_{2}(s) & \left(\int_{0}^{s}\left[e_{2}(s)+e_{3}(x)+K_{0}(s, x)|\phi(x)|^{\sigma}\right] d x\right. \\
& +\sum_{i=1}^{n^{2}-1} \sum_{\ell=0}^{i-1}\left|K_{i}^{(\ell)}(s)\right| \beta \\
& \left.\left.+\sum_{i=1}^{n^{2}-1} \int_{0}^{s}\left|\frac{\partial^{i}}{\partial x^{i}} K_{i}^{(i)}(s-x)\right||\phi(x)| d x\right)\right] w(s) d s .
\end{aligned}
$$

Let,

$$
\begin{aligned}
& h(t)=\sum_{j=1}^{n^{2}}\left(C_{j}+\left|\alpha_{j}\right|\right)\left|\phi_{j}(t)\right| \\
&+\sum_{j, k=1}^{n^{2}}\left|\zeta^{j k}\right|\left|\phi_{j}(t)\right| \int_{0}^{t}\left|\overline{\phi_{k}^{+}(s)}\right|\left[r _ { 2 } ( s ) \left[\int_{0}^{s}\left[e_{2}(s)+e_{3}(x)\right] d x\right.\right. \\
&\left.\left.+\sum_{i=1}^{n^{2}-1} \sum_{\ell=0}^{i=1}\left|K_{i}^{(\ell)}(s)\right| \beta\right]\right] w(s) d s .
\end{aligned}
$$

Then,

$$
\begin{aligned}
& |\phi(t)| \\
& \leq h(t)+\sum_{j, k=1}^{n^{2}}\left|\zeta^{j k}\right|\left|\phi_{j}(t)\right| \\
& \times \int_{0}^{t}\left|\overline{\phi_{k}^{+}(s)}\right|\left[r_{1}(s)|\phi(s)|^{\sigma}\right. \\
& +r_{2}(s)\left[\int_{0}^{s} k_{0}(s, x)|\phi(x)|^{\sigma} d x\right. \\
& \left.\left.\quad+\sum_{i=1}^{n^{2}-1} \int_{0}^{s}\left|\frac{\partial^{i}}{\partial x^{i}} K_{i}^{(i)}(s-x)\right||\phi(x)| d x\right]\right] w(s) d s .
\end{aligned}
$$

By hypothesis, there exist positive constants $\xi_{1}$ and $\xi_{2}$ such that,

$$
\left|\phi_{j}(t)\right| \leq \xi_{1}, \quad\left|\phi_{k}^{+}(t)\right| \leq \xi_{2} \quad \forall j, k=1,2, \ldots, n^{2},
$$

and from conditions (i), (ii), and (iii), it follows that $h(t)$ is bounded on $[0, b)$, that is, there exists a positive constant $\xi_{3}$ such that $h(t) \leq \xi_{3}$ for all $t \in[0, b)$. 
Then,

$$
\begin{aligned}
|\phi(t)| \leq \xi_{3}+n^{2} \xi_{1} \xi_{2}[ & \int_{0}^{t} r_{1}(s)|\phi(s)|^{\sigma} w(s) d s \\
& +\int_{0}^{t} r_{2}(s)\left(\int_{0}^{s} k_{0}(s, x)|\phi(x)|^{\sigma} d x\right) w(s) d s \\
& \left.+\sum_{i=1}^{n^{2}-1} \int_{0}^{t}\left(r_{2}(s) \int_{0}^{s}\left|\frac{\partial^{i}}{\partial x^{i}} K_{i}(s-x)\right||\phi(x)| d x\right) w(s) d s\right] .
\end{aligned}
$$

Applying Corollary 3.8 with $f_{1}(x)=0$, we have

$$
\begin{aligned}
&|\phi(t)| \leq \exp \left(\int_{0}^{t} r_{2}(s)\left(\sum_{i=1}^{n^{2}-1} \int_{0}^{s}\left|\frac{\partial^{i}}{\partial x^{i}} K_{i}(s-x)\right| d x\right) w(s) d s\right) \\
& \times\left\{\left[\xi_{3}^{(1-\sigma)}+(1-\sigma)\right.\right. \\
& \times \int_{0}^{t}\left(r_{1}(s)+\int_{0}^{s} r_{2}(x) k_{0}(s, x) d x\right) \\
& \times \exp (1-\sigma)\left(\int_{0}^{t}\left(r_{2}(\tau) \sum_{i=1}^{n^{2}-1} \int_{0}^{\tau}\left|\frac{\partial^{i}}{\partial x^{i}} K_{i}(s-x)\right| d x\right)\right. \\
&\left.\times w(\tau) d \tau) w(s) d s]^{1 /(1-\sigma)}\right\}
\end{aligned}
$$

Hence, from conditions (i), (ii), and (iii), it follows that $\phi(s)$ is bounded on $[0, b)$.

THEOREM 4.4. Suppose that $S(\tau) \cup S\left(\tau^{+}\right) \subset L_{w}^{2}(a, b), r_{2}(s)$ is bounded on $[0, b)$, and the following conditions are satisfied:

(i) $e_{1}(s) \in L_{w}^{2}(0, b)$ and $r_{1}(s) \in L_{w}^{2 /(1-\sigma)}(0, b)$ for all $s \in[0, b)$,

(ii) $\int_{0}^{t}\left(\int_{0}^{s}\left[e_{2}(s)+e_{3}(x)\right] d x\right)^{2} w(s) d s<\infty$,

(iii) $\int_{0}^{t}\left(\sum_{i=1}^{n^{2}-1} \sum_{\ell=0}^{i-1}\left|K_{i}^{(\ell)}(s)\right|\right)^{2} w(s) d s<\infty$,

(iv) $\left[\int_{0}^{s} w^{\sigma /(\sigma-2)} K_{0}^{2 /(2-\sigma)}(s, x) d x\right]^{(2-\sigma) / 2}<\infty$,

(v) $\left[\int_{0}^{s} w^{-1}\left|\left(\partial^{i} / \partial x^{i}\right) K_{i}(s-x)\right|^{2} d x\right]^{1 / 2} w(s) d s<\infty$.

Then all solutions of (1.2) are in $L_{w}^{2}(0, b)$. 
Proof. For $0 \leq \sigma<1$, the proof is the same up to (4.18). Applying the Cauchy-Schwartz inequality to the integrals in (4.18), we have that

$$
\begin{aligned}
& \int_{0}^{t}\left|\overline{\phi_{j}^{+}(s)}\right| r_{1}(s)|\phi(s)|^{\sigma} w(s) d s \\
& \quad \leq\left(\int_{0}^{t}|\phi(s)|^{2} w(s) d s\right)^{\sigma / 2}\left(\int_{0}^{t}\left|\overline{\phi_{k}^{+}(s)} r_{1}(s)\right|^{\mu} w(s) d s\right)^{1 / \mu}, \\
& \int_{0}^{s} K_{0}(s \cdot x)\left|\overline{\phi_{k}^{+}(s)}\right|^{\sigma} d x \\
& \quad \leq\left(\int_{0}^{s}|\phi(x)|^{2} w(x) d x\right)^{\sigma / 2}\left(\int_{0}^{s} w^{1-\mu} K_{0}^{\mu}(s, x) d x\right)^{1 / \mu}, \\
& \int_{0}^{s}\left|\frac{\partial^{i}}{\partial x^{i}} K_{i}(s-x) \phi(x)\right| d x \\
& \quad \leq\left(\int_{0}^{s}|\phi(x)|^{2} w(x) d x\right)^{1 / 2}\left(\int_{0}^{s} w^{-1}\left|\frac{\partial^{i}}{\partial x^{i}} K_{i}(s-x)\right|^{2} d x\right)^{1 / 2},
\end{aligned}
$$

where $\mu=2 /(2-\sigma)$. Since $\phi_{k}^{+}(s) \in L_{w}^{2}(0, b)$ (see Lemma 3.3), $k=1, \ldots, n^{2}$, and $r_{1}(s) \in L_{w}^{2 /(1-\sigma)}(0, b)$ by hypothesis, we have $\phi_{k}^{+} r_{1} \in L_{w}^{\mu}(0, b), k=1,2, \ldots, n^{2}$. Using this fact and (4.18) in (4.16), we obtain

$$
\begin{aligned}
|\phi(t)| \leq h(t)+ & \sum_{j, k=1}^{n^{2}}\left|\zeta^{j k}\right|\left|\phi_{j}(t)\right| \\
\times & {\left[\xi_{0}\left(\int_{0}^{t}|\phi(s)|^{2} w(s) d s\right)^{\sigma / 2}\right.} \\
& +\xi_{1}\left(\int_{0}^{t}\left|\overline{\phi_{k}^{+}(s)}\right| r_{2}(s)\left(\int_{0}^{s}|\phi(x)|^{2} w(x) d x\right)^{\sigma / 2} w(s) d s\right) \\
& \left.+n^{2} \xi_{2}\left(\int_{0}^{t}\left|\overline{\phi_{k}^{+}(s)}\right| r_{2}(s)\left(\int_{0}^{s}|\phi(x)|^{2} w(x) d x\right)^{1 / 2} w(s) d s\right)\right],
\end{aligned}
$$

where

$$
\begin{aligned}
& \xi_{0}=\left(\int_{0}^{t}\left|\overline{\phi_{k}^{+}(s)} r_{1}(s)\right|^{\mu} w(s) d s\right)^{1 / \mu}, \\
& \xi_{1}=\left(\int_{0}^{t} w^{1-\mu} K_{0}^{\mu}(s, x) d x\right)^{1 / \mu}, \\
& \xi_{2}=\left(\int_{0}^{t} w^{-1}\left|\frac{\partial^{i}}{\partial x^{i}} K_{i}(s-x)\right|^{2} d x\right)^{1 / 2}, \text { for } i=1,2, \ldots, n^{2} .
\end{aligned}
$$


Applying the Cauchy-Schwartz inequality again to the integrals in (4.25) and squaring both sides, we have

$$
\begin{aligned}
& |\phi(t)|^{2} \\
& \leq 2 h^{2}(t)+4 \sum_{j, k=1}^{n^{2}}\left|\zeta^{j k}\right|^{2}\left|\phi_{j}(t)\right|^{2} \\
& \times\left[\xi_{0}^{2}\left(\int_{0}^{t}|\phi(s)|^{2} w(s) d s\right)^{\sigma}\right. \\
& +\xi_{1}^{2}\left(\int_{0}^{t}\left|\overline{\phi_{k}^{+}(s)}\right|^{2} w(s) d s\right)\left(\int_{0}^{t} r_{2}^{2}\left(\int_{0}^{s}|\phi(x)|^{2} w(x) d x\right)^{\sigma}\right) \\
& +n^{4} \xi_{0}^{2}\left(\int_{0}^{t}\left|\overline{\phi_{k}^{+}(s)}\right|^{2} w(s) d s\right)\left(\int_{0}^{t} r_{2}^{2}\left(\int_{0}^{s}|\phi(x)|^{2} w(x) d x\right)^{\sigma}\right. \\
&
\end{aligned}
$$

Let

$$
\begin{aligned}
u(t) & =\int_{0}^{t}|\phi(s)|^{2} w(s) d s, \\
\xi_{3} & =\left(\int_{0}^{t}\left|\overline{\phi_{k}^{+}(s)}\right|^{2} w(s) d s\right)^{1 / 2}, \quad j=1,2, \ldots, n^{2},
\end{aligned}
$$

and integrate (4.27), to obtain

$$
\begin{aligned}
u(t) \leq & 2 \int_{0}^{t} h^{2}(s) w(s) d s \\
& +4 \xi_{0}^{2} \sum_{j, k=1}^{n^{2}}\left|\zeta^{j k}\right|^{2} \int_{0}^{t}\left|\phi_{j}(s)\right|^{2} u^{\sigma}(s) w(s) d s \\
& +4 \xi_{1}^{2} \xi_{3}^{2} \sum_{j, k=1}^{n^{2}}\left|\zeta^{j k}\right|^{2} \int_{0}^{t}\left|\phi_{j}(s)\right|^{2}\left(\int_{0}^{s} r_{2}^{2}(x) u^{\sigma}(x) w(x) d x\right) w(s) d s \\
& +4 n^{4} \xi_{2}^{2} \xi_{3}^{2} \sum_{j, k=1}^{n^{2}}\left|\zeta^{j k}\right|^{2} \int_{0}^{t}\left|\phi_{j}(s)\right|^{2}\left(\int_{0}^{s} r_{2}^{2}(x) u(x) w(x) d x\right) w(s) d s .
\end{aligned}
$$

From our assumptions and conditions (ii) and (iii), it follows that the integral $\int_{0}^{t} h^{2}(s) w(s) d s$ is finite, that is, there exists a positive constant $\xi_{4}$ such that $\|h(t)\|_{L_{w}^{2}(0, b)} \leq \xi_{4}$ for all $t \in[0, b)$. Applying Corollary 3.8 with $f_{1}(x)=0$, we 
obtain

$$
\begin{aligned}
& u(t) \leq \exp \left(\int_{0}^{t}\left(4 n^{4} \xi_{2}^{2} \xi_{3}^{2} \sum_{j, k=1}^{n^{2}}\left|\zeta^{j k}\right|^{2}\left|\phi_{j}(s)\right|^{2} \int_{0}^{s} r_{2}^{2}(x) w(x) d x\right) w(s) d s\right) \\
& \times {\left[\xi_{4}^{(1-\sigma)}+(1-\sigma)\right.} \\
& \times \int_{0}^{t} 4 \sum_{j, k=1}^{n^{2}}\left|\zeta^{j k}\right|^{2}\left|\phi_{j}(s)\right|^{2}\left(\xi_{0}^{2}+\xi_{1}^{2} \xi_{3}^{2} \int_{0}^{s} r_{2}^{2}(x) w(x) d x\right) \\
& \times \exp \left((1-\sigma) \int_{0}^{s}\left[4 n^{4} \xi_{2}^{2} \xi_{3}^{2} \sum_{j, k=1}^{n^{2}}\left|\zeta^{j k}\right|^{2}\left|\phi_{j}(x)\right|^{2} \int_{0}^{x} r_{2}^{2}(\tau) w(\tau) d \tau\right]\right. \\
&\times w(x) d x) w(s) d s]^{1 /(1-\sigma)}
\end{aligned}
$$

Since $\phi_{j}(t) \in L_{w}^{2}(0, b), j=1,2, \ldots, n^{2}$, and $r_{2}(t)$ is bounded on $[0, b)$, then $\phi(t) \in L_{w}^{2}(0, b)$ and hence the result.

COROLLARY 4.5. Suppose that $S(\tau) \cup S\left(\tau^{+}\right) \subset L_{w}^{2}(0, b) \cap L^{\infty}(0, b)$ and the following conditions are satisfied:

(i) $e_{1}(s) \in L_{w}^{2}(0, b)$ and $r_{1}(s) \in L_{w}^{p}(0, b)$ for any $p, 1 \leq p \leq 2 /(1-\sigma)$,

(ii) $r_{2}(s)$ and $K_{i}^{(\ell)}(s) \in L_{w}^{2}(0, b) \cap L^{\infty}(0, b)$ for $\ell=0, \ldots, i-1 ; i=1, \ldots, n^{2}-$ 1 ,

(iii) $\int_{0}^{t}\left(\int_{0}^{s}\left[e_{2}(s)+e_{3}(x)\right] d x\right)^{2} w(s) d s<\infty$,

(iv) $\left(\int_{0}^{s} w^{\sigma /(\sigma-2)} K_{0}^{2 /(2-\sigma)}(s, x) d x\right)^{(2-\sigma) / 2}<\infty, 0 \leq \sigma<1$,

(v) $\left[\int_{0}^{s} w^{-1}\left|\left(\partial^{i} / \partial x^{i}\right) K_{i}(s-x)\right|^{2} d x\right]^{1 / 2}<\infty, i=1, \ldots, n^{2}-1$.

Then all solutions of (1.2) belong to $L_{w}^{2}(0, b) \cap L^{\infty}(0, b)$.

Proof. The proof follows from Theorems 4.3 and 4.4. We refer to [6, 7, 8, 11] for more details.

\section{REFERENCES}

[1] R. P. Agarwal, A note on Grace and Lalli's paper, J. Math. Anal. Appl. 86 (1982), no. 2, 471-475.

[2] D. E. Edmunds and W. D. Evans, Spectral Theory and Differential Operators, Oxford Mathematical Monographs, Oxford University Press, New York, 1987.

[3] W. N. Everitt and D. Race, Some remarks on linear ordinary quasidifferential expressions, Proc. London Math. Soc. (3) 54 (1987), no. 2, 300-320.

[4] W. N. Everitt and A. Zettl, The number of integrable-square solutions of products of differential expressions, Proc. Roy. Soc. Edinburgh Sect. A 76 (1977), 215226.

[5] S. R. Grace and B. S. Lalli, Asymptotic behaviour of certain second order integrodifferential equations, J. Math. Anal. Appl. 76 (1980), no. 1, 84-90.

[6] S. E. Ibrahim, Boundedness for solutions of general ordinary quasi-differential equations, J. Egyptian Math. Soc. 2 (1994), 33-44. 
[7] _ On $L_{w}^{2}$-quasi-derivatives for solutions of perturbed general quasidifferential equations, Czechoslovak Math. J. 49(124) (1999), no. 4, 877890.

[8] J. S. W. Wong, Square integrable solutions of perturbed linear differential equations, Proc. Roy. Soc. Edinburgh Sect. A 73 (1975), 251-254.

[9] E. H. Yang, On asymptotic behaviour of certain integro-differential equations, Proc. Amer. Math. Soc. 90 (1984), no. 2, 271-276.

[10] _ Asymptotic behaviour of certain second order integro-differential equations, J. Math. Anal. Appl. 106 (1985), no. 1, 132-139.

[11] A. Zettl, Square integrable solutions of $L y=f(t, y)$, Proc. Amer. Math. Soc. 26 (1970), 635-639.

[12] _ Deficiency indices of polynomials in symmetric differential expressions. II, Proc. Roy. Soc. Edinburgh Sect. A 73 (1975), no. 20, 301-306.

[13]_ Formally self-adjoint quasi-differential operators, Rocky Mountain J. Math. 5 (1975), 453-474.

[14] _ The limit-point and limit-circle cases for polynomials in a differential operator, Proc. Roy. Soc. Edinburgh Sect. A 72 (1975), no. 3, 219-224.

[15] _ Perturbation of the limit circle case, Quart. J. Math. Oxford Ser. (2) 26 (1975), no. 103, 355-360.

Sobhy El-Sayed Ibrahim: Department of Mathematics, Faculty of Science, Benha University, Benha 13518, Kalubia, Egypt

E-mail address: abomohamed@hotmai 1 .com 


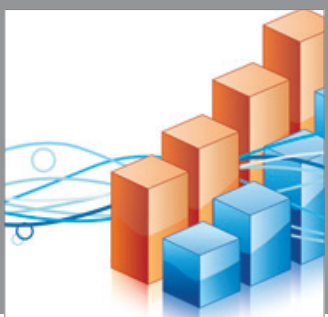

Advances in

Operations Research

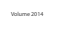

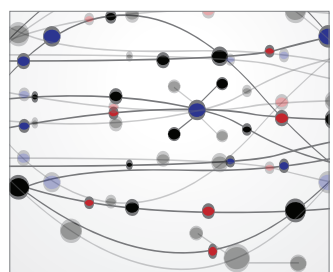

\section{The Scientific} World Journal
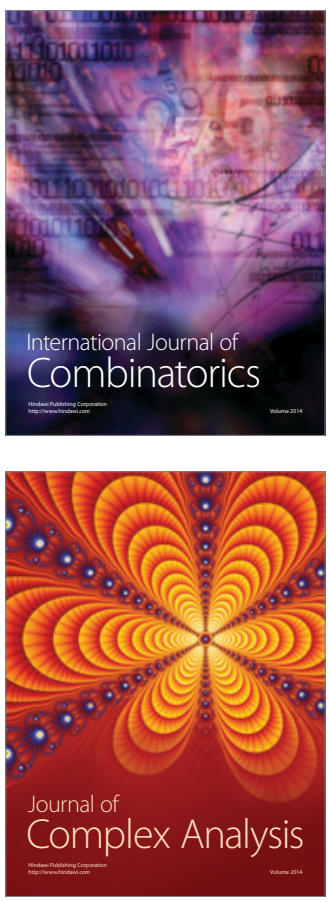

International Journal of

Mathematics and

Mathematical

Sciences
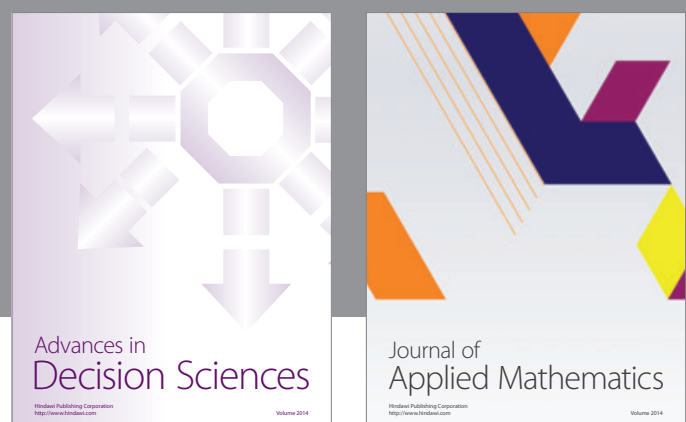

Journal of

Applied Mathematics
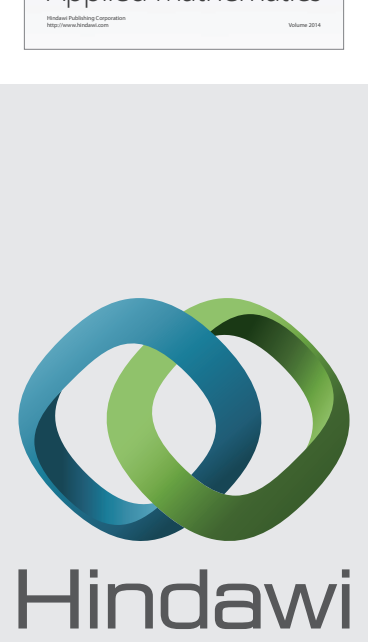

Submit your manuscripts at http://www.hindawi.com
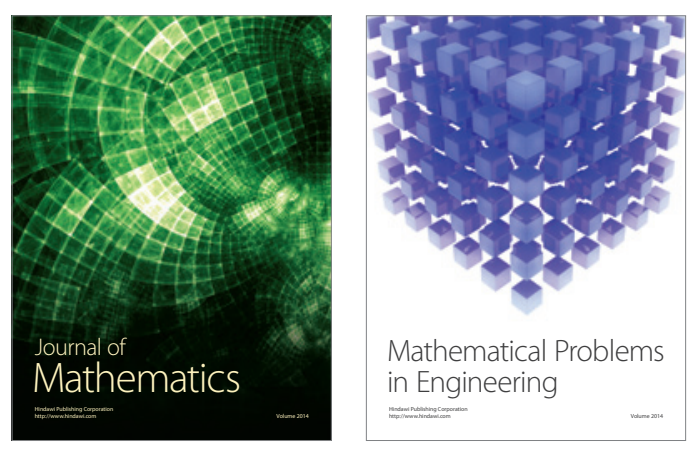

Mathematical Problems in Engineering
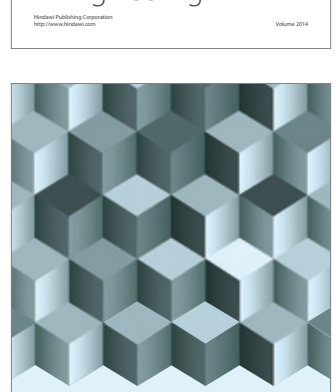

Journal of

Function Spaces
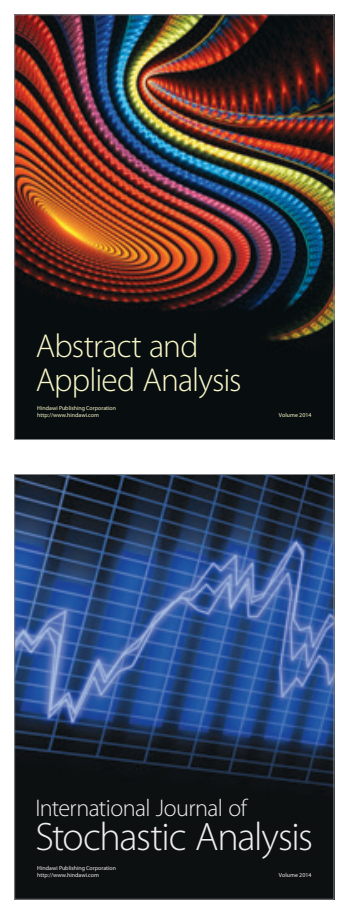

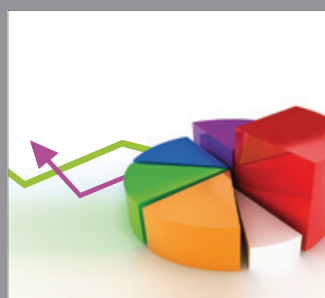

ournal of

Probability and Statistics

Promensencen
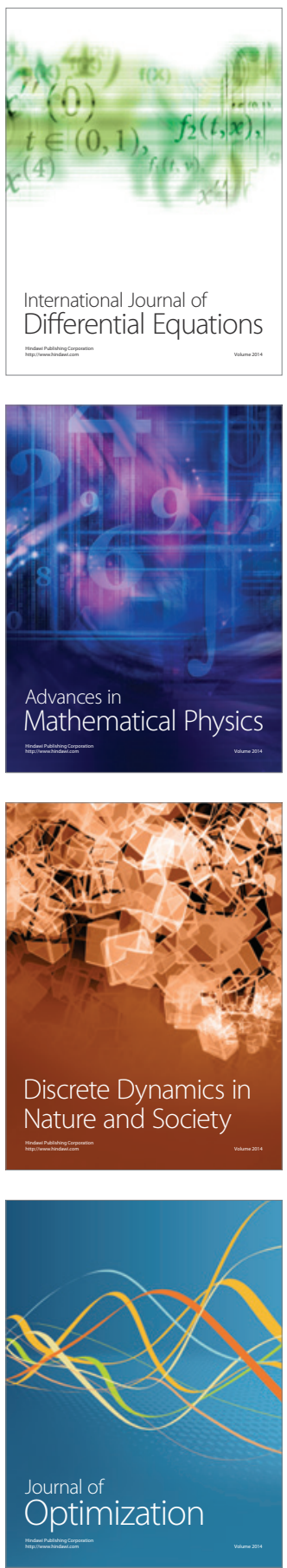